\title{
聚集诱导发光探针用于细胞缺血再灌注诱导过氧化氢成像研究
}

\author{
李维贾旭郭振波姜文婷 \\ 张平竹魏 超* 李小六* \\ (河北大学化学与环境科学学院 河北省化学生物学重点实验室 药物化学与分子诊断教育部重点实验室 \\ 河北保定 071002)
}

\begin{abstract}
摘要 过氧化氢是一种重要的内源性信号分子, 参与调控多种生理和病理过程. 缺血再灌注会诱导产生大量内源性过 氧化氢, 对细胞和组织造成严重损伤. 聚集诱导发光探针能够规避常规荧光团浓度过大所引起的聚集荧光淬灭的问题. 以 4-乙烯吡啶修饰的四苯乙烯为荧光基团, 苯硼酸为过氧化氢识别基团, 设计合成了一种具有聚集诱导发光性质的长 波长过氧化氢荧光探针. 光谱测试结果表明, 探针对过氧化氢具有较好的选择性和较高的灵敏度, 最低检测限为 $6.9 \times$ $10^{-8} \mathrm{~mol} / \mathrm{L}$. 共聚焦成像结果表明, 探针具有较好的细胞通透性, 可用于糖氧剥夺再灌注诱导 HeLa 细胞和脂多糖诱导 斑马鱼内源性过氧化氢生成研究.
\end{abstract}

关键词＼cjkstart过氧化氢；氧糖剥离再灌注；聚集诱导发光；荧光探针；生物成像

\section{Imaging of Hydrogen Peroxide During the Ischemia Reperfusion Process in Living Cells with An Aggregation Induced-Emission Probe}

\author{
Li, Wei Jia, Xu Guo, Zhenbo Jiang, Wenting \\ Zhang, Pingzhu Wei, Chao* Li, Xiaoliu*
}

(Key Laboratory of Chemical Biology of Hebei Province, Key Laboratory of Medicinal Chemistry and Molecular Diagnosis of Ministry of Education, College of Chemistry and Environmental Science, Hebei University, Baoding, Hebei 071002)

\begin{abstract}
As an important endogenous signaling molecule, hydrogen peroxide $\left(\mathrm{H}_{2} \mathrm{O}_{2}\right)$ is involved in regulating many physiological and pathological processes. Ischemia-reperfusion can induce the production of large amount of endogenous hydrogen peroxide, which can cause seriously damage to cells and tissues. The fluorescent probe with aggregation induced emission can avoid the shortage of the aggregation caused quenching of conventional fluorophores. $\mathrm{A}_{2} \mathrm{O}_{2}$ fluorescent probe with aggregation induced emission properties was designed and synthesized by using 4-vinylpyridinyl modified tetraphenylethylene as the fluorophore and phenylboronic acid as the $\mathrm{H}_{2} \mathrm{O}_{2}$ sensing group. The structure of the probe was characterized by NMR and HRMS. The recognition behaviors of the probe to $\mathrm{H}_{2} \mathrm{O}_{2}$ were investigated by the UV-Vis absorption and fluorescence spectra, and the results exhibit its good selectivity and high sensitivity to $\mathrm{H}_{2} \mathrm{O}_{2}$. The fluorescence off-on enhancement was $c a$. 100-fold and the detection limit was $6.9 \times 10^{-8} \mathrm{~mol} / \mathrm{L}$. The reaction of the probe and $\mathrm{H}_{2} \mathrm{O}_{2}$ resulted in the $\mathrm{H}_{2} \mathrm{O}_{2}$-mediated oxidation of phenylboronic acid, followed by hydrolysis and 1,6-elimination of $p$-quinone-methide to generate $(E)-4-(4-(2,2-b i s(4-$ methoxyphenyl)-1-phenylvinyl)styryl)pyridine (TPE-Py), which was confirmed by ${ }^{1} \mathrm{H}$ NMR. The results of confocal imaging indicated that the probe was cell-permeable and capable of visualization of endogenous $\mathrm{H}_{2} \mathrm{O}_{2}$ in oxygen glucose deprivation/reoxygenation (OGD/R) model HeLa cells and lipopolysaccharide-treated zebrafish.

Keywords hydrogen peroxide; oxygen glucose deprivation/reoxygenation (OGD/R); aggregation induced-emission; fluorescent probe; bioimaging
\end{abstract}

活性氧物种(Reactive oxygen species, ROS)是生命

体有氧代谢所产生的一类具有氧化性的活性小分子，主

\footnotetext{
* Corresponding authors. E-mail: weichao@hbu.edu.cn; lixl@hbu.cn

Received March 16, 2020; revised April 30, 2020; published online May 15, 2020.

Project supported by the Natural Science Foundation of Hebei Province (No. B2018201234) and the Colleges and Universities Science Technology Research Project of Hebei Province (No. QN2017015).

河北省自然科学基金(No. B2018201234)和河北省高等学校科学技术研究(No. QN2017015)资助项目.
} 
要包括超氧阴离子 $\left(\mathrm{O}^{2-}\right)$ 、单线态氧 $\left({ }^{1} \mathrm{O}_{2}\right)$ 、羟基自由基 $(\cdot \mathrm{OH})$ 和过氧化氢 $\left(\mathrm{H}_{2} \mathrm{O}_{2}\right)$ 等 ${ }^{[1]}$. $\mathrm{H}_{2} \mathrm{O}_{2}$ 不仅是 $\mathrm{ROS}$ 的关键 组成部分, 更是一种重要的内源性信号分子. 细胞内 $\mathrm{H}_{2} \mathrm{O}_{2}$ 通常由线粒体呼吸链电子传递异常或烟酰胺腺嘌 呤二核苷酸磷酸氧化酶过度激活所产生, 其在细胞分 化、增殖、调亡、信号转导和机体免疫等多种生理过程 中发挥重要作用 ${ }^{[2-3]}$. 研究表明, 内源性 $\mathrm{H}_{2} \mathrm{O}_{2}$ 浓度过高 会诱导细胞发生氧化应激, 破坏蛋白质, 从而导致多种 严重疾病的发生, 如心脏病、糖尿病、神经退行性疾病 和癌症等 ${ }^{[4-6]}$. 另外, 缺血再灌注是组织缺血或缺氧一段 时间后供血恢复的临床高发现象. 在外科手术、器官移 植、创伤性休克和血栓等多种血液循环障碍时, 均会出 现缺血后再灌注损伤. 缺血再灌注会诱导细胞产生大量 $\mathrm{H}_{2} \mathrm{O}_{2}$, 对组织器官造成损伤 ${ }^{[7]}$. 因此, 基于 $\mathrm{H}_{2} \mathrm{O}_{2}$ 在生物 化学和临床医学的重要意义, 开发能够原位检测 $\mathrm{H}_{2} \mathrm{O}_{2}$ 水平的可视化工具具有重要意义.

传统的 $\mathrm{H}_{2} \mathrm{O}_{2}$ 检测方法主要有比色法 ${ }^{[8]}$ 、紫外可见分 光光度法 ${ }^{[9]}$ 和电化学法 ${ }^{[10-11]}$ 等, 这些都不适合生物样本 中实时动态观测 $\mathrm{H}_{2} \mathrm{O}_{2}$. 苂光方法具有操作简便、灵敏性 高、选择性可调及适合于活体成像等优点, 是检测内源 性 $\mathrm{H}_{2} \mathrm{O}_{2}$ 的首选方法. 基于 $\mathrm{H}_{2} \mathrm{O}_{2}$ 分子的氧化性和亲核性, 多种类型的 $\mathrm{H}_{2} \mathrm{O}_{2}$ 苂光探针得到开发 ${ }^{[12-15]}$, 其识别基团 主要有苯嗍酸 ${ }^{[16-25]}$ 和芳香邻二酮 ${ }^{[26-29]}$ 及其衍生物 (Scheme 1). 2004 年, Chang 等 ${ }^{[16]}$ 报道了首例硼酸基 $\mathrm{H}_{2} \mathrm{O}_{2}$ 荧光探针, 该探针以苂光素为荧光团, 频哪醇硼酸酯为 识别基团. 2011 年, Nagano 等 ${ }^{[26]}$ 开发了以 4-硝基苯偶酰 为识别基团的 $\mathrm{H}_{2} \mathrm{O}_{2}$ 苂光探针, 该探针通过调控硝基的
光诱导电子转移猝灭能力来实现对 $\mathrm{H}_{2} \mathrm{O}_{2}$ 的荧光响应. 2016 年, 唐波课题组 ${ }^{[28]}$ 将 $\alpha$-酮酰胺与分子内电荷转移 机理相结合, 设计合成了一例近红外 $\mathrm{H}_{2} \mathrm{O}_{2}$ 荧光探针. 上 述传统探针的荧光团具有较大的共轭结构, 其在大浓度 使用时，荧光团会产生 $\pi-\pi$ 堆积，造成探针聚集荧光淬 灭.

2001 年, 唐本忠等 ${ }^{[30]}$ 创新性发现了基于分子内运 动受限原理的 “聚集诱导发光” (AIE)现象. 发展 AIE 苂光探针有利于规避常规荧光团浓度过大所引起的聚 集荧光淬灭的问题 ${ }^{[31]}$. 唐波课题组 ${ }^{[32]}$ 在四苯乙烯分子 上直接修饰硼酸频哪醇酯, 设计合成了双硼酸基 AIE 苂 光探针 AIE-1 (Scheme 2). 张德清课题组 ${ }^{[33]}$ 在四苯乙烯 结构中引入吡啶基团来增加共轭结构, 设计合成了硼酸 基 AIE 荧光探针 AIE-2 (Scheme 2). 上述 AIE 探针实现 了 $\mathrm{H}_{2} \mathrm{O}_{2}$ 的选择性检测, 但较短的荧光发射波长不利于 其在生物体内的广泛使用.

本工作设计合成了一种具有 $\mathrm{AIE}$ 性质的长荧光发 射波长的 $\mathrm{H}_{2} \mathrm{O}_{2}$ 探针 1. 探针以 4-乙烯基吡啶修饰的四苯 乙烯为荧光团, 通过增大荧光团的共轭结构, 将探针的 荧光发射扩展至黄绿光区. 探针以苯硼酸为识别基团, 与 $\mathrm{H}_{2} \mathrm{O}_{2}$ 反应后苂光增强, 实现了 $\mathrm{H}_{2} \mathrm{O}_{2}$ 打开型检测 (Scheme 2). 探究了探针对 $\mathrm{H}_{2} \mathrm{O}_{2}$ 响应的灵敏性和选择 性. 利用苂光共聚焦显微成像, 实现了细胞和斑马鱼内 $\mathrm{H}_{2} \mathrm{O}_{2}$ 的苂光成像. 根据文献构建了细胞氧糖剥离再灌 注 $(\mathrm{OGD} / \mathrm{R})$ 模型来模拟在体的缺血缺氧状态, 研究了此 模型中细胞内源 $\mathrm{H}_{2} \mathrm{O}_{2}$ 的变化.

A

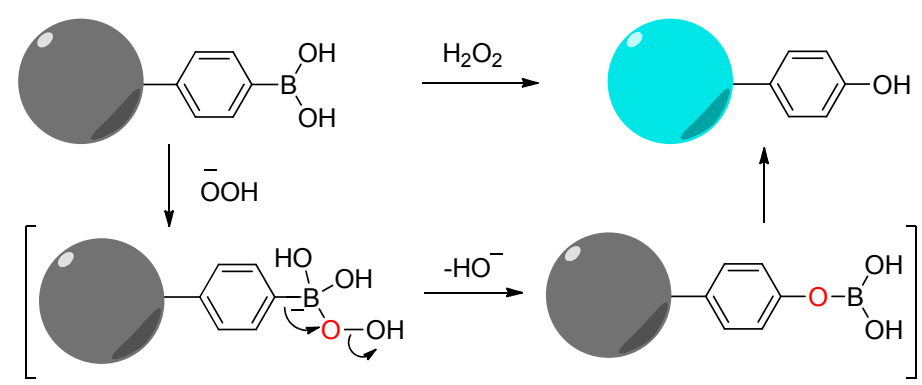

B

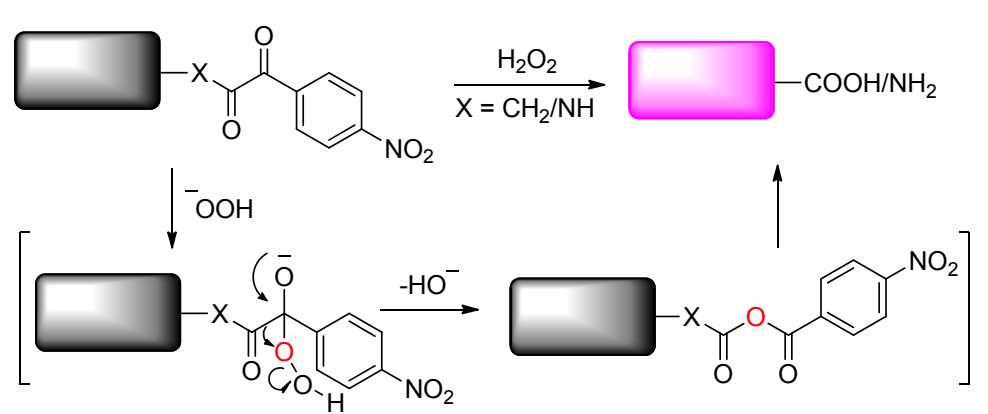

图式 1 以苯嗍酸(A)和芳香邻二酮衍生物(B)为识别基团的苂光探针与 $\mathrm{H}_{2} \mathrm{O}_{2}$ 识别示意图

Scheme 1 Sensing schematic diagram of fluorescence $\mathrm{H}_{2} \mathrm{O}_{2}$ probes using benzoic acid (A) and benzoyl derivatives (B) as sensing groups 


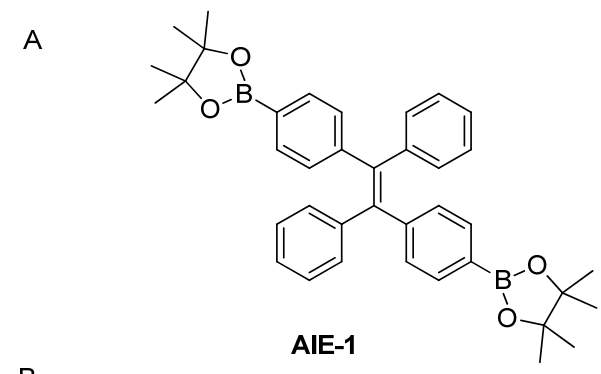<smiles>COc1ccc(C(=C(c2ccc(OC)cc2)c2ccc(-c3cc[n+](Cc4ccc(B5OC(C)(C)C(C)(C)O5)cc4)cc3)cc2)c2ccccc2)cc1</smiles>

B<smiles>COc1ccc(C(=C(c2ccc(C=Cc3ccncc3)cc2)c2ccc(OC)cc2)c2ccccc2)cc1</smiles>

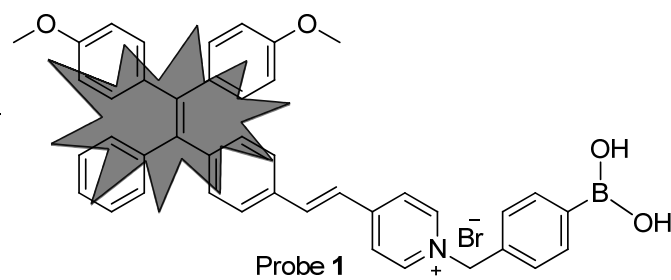<smiles>C=C1C=CC(=O)C=C1C(C)O</smiles>

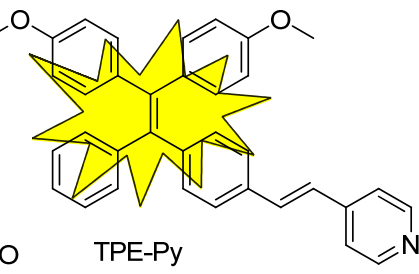

图式 2 (A) 已报道具有 AIE 性质的 $\mathrm{H}_{2} \mathrm{O}_{2}$ 苂光探针 AIE-1 和 AIE-2 以及(B)探针 $\mathbf{1}$ 的合成及其与 $\mathrm{H}_{2} \mathrm{O}_{2}$ 反应示意图 Scheme 2 (A) Reported $\mathrm{H}_{2} \mathrm{O}_{2}$ fluorescent probe AIE-1 and AIE-2 with the properties of AIE, and (B) the synthesis and sensing mechanism with $\mathrm{H}_{2} \mathrm{O}_{2}$ of probe 1

\section{1 结果与讨论}

\section{1 探针光谱性质与对过氧化氢的响应}

\subsection{1 探针光谱性质}

为了探究探针是否具有 AIE 特性, 在二甲基亚砜 (DMSO) $/ \mathrm{H}_{2} \mathrm{O}$ 体系中测试了 TPE-Py、探针 1 及其与 $\mathrm{H}_{2} \mathrm{O}_{2}$ 反应的荧光发射光谱. 如图 $1 \mathrm{~A}$ 所示, 随着溶液中水含 量的增加, TPE-Py 的荧光逐渐强度, 说明 TPE-Py 分子 发生了聚集, 限制了分子的运动, 具有聚集诱导发光能 力. 探针 $\mathbf{1}$ 与 $\mathrm{H}_{2} \mathrm{O}_{2}$ 反应的苂光光谱同样表明, 探针 $\mathbf{1}$ 具 有 AIE 能力(图 1B). 探针以 4-乙烯基吡啶修饰的四苯乙 烯为荧光团, 通过增大荧光团的共轭结构, 将探针的最 大苂光发射波长延长至 $545 \mathrm{~nm}$. 与 20 equiv. $\mathrm{H}_{2} \mathrm{O}_{2}$ 反应 后，探针荧光增强高达 100 倍，远高于其它 AIE 型苂光 探 针 $^{[32-33]}$.

\subsection{2 探针对过氧化氢的响应}

为了探究探针的检测灵敏性, 进行了苂光滴定实 验. 如图 2A 所示, 随着加入 $\mathrm{H}_{2} \mathrm{O}_{2}$ 浓度逐渐增大, 探针 的荧光逐渐增强. 以 $545 \mathrm{~nm}$ 处探针的荧光相对强度 $I / I_{0}$ 对 $\mathrm{H}_{2} \mathrm{O}_{2}$ 浓度作图, 发现在 $\mathrm{H}_{2} \mathrm{O}_{2}$ 浓度 $75 \sim 250 \mu \mathrm{mol} / \mathrm{L}$ 范 围内线性关系良好(图 2B), 按照 $3 \sigma / k$ 方法计算探针的检 测极限为 $6.9 \times 10^{-8} \mathrm{~mol} / \mathrm{L}$. 表明探针能够对 $\mathrm{H}_{2} \mathrm{O}_{2}$ 高灵 敏性检测.

生命体是一个复杂的系统, 含有多种潜在干扰物 质. 为了验证探针的检测选择性, 测试了探针分别与多 种金属阳离子 $\left(\mathrm{Cu}^{2+} 、 \mathrm{Mg}^{2+} 、 \mathrm{Zn}^{2+} 、 \mathrm{Fe}^{3+}\right)$ 、阴离子 $\left(\mathrm{SO}_{4}^{2-}\right.$ 、 $\left.\mathrm{NO}_{3}^{-} 、 \mathrm{NO}_{2}^{-} 、 \mathrm{HSO}_{3}^{-}\right)$和具有氧化还原能力的活性分子 $\left(\mathrm{NaClO} 、 \mathrm{KO}_{2} 、 \mathrm{ONOO}^{-} 、 t\right.$-BuOOH、NO、GSH、Cys、
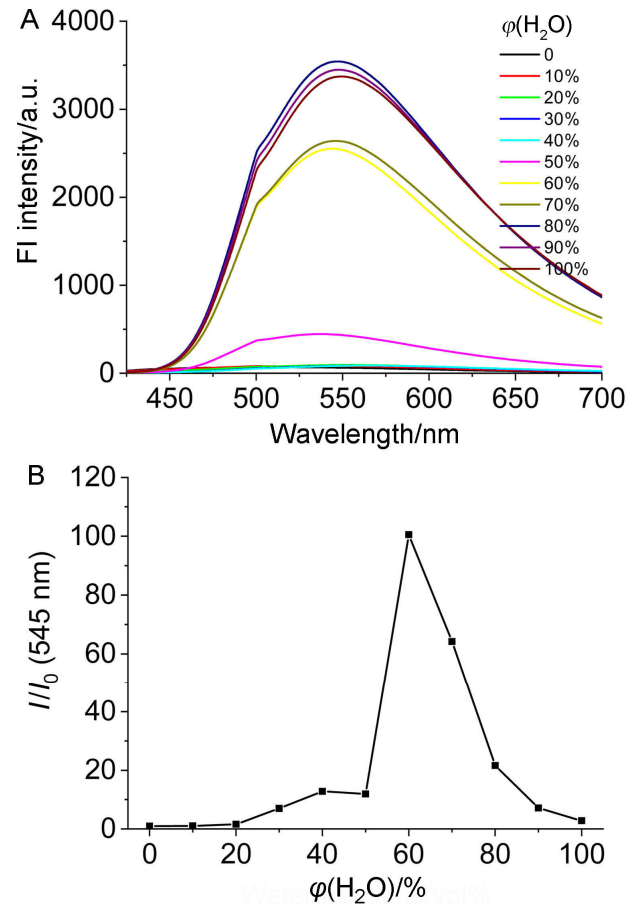

图 1 (A) 探针 TPE-Py 在 $\mathrm{DMSO} / \mathrm{H}_{2} \mathrm{O}$ 体系中的苂光发射光谱 和(B)探针 $1(10 \mu \mathrm{mol} / \mathrm{L})$ 与 $\mathrm{H}_{2} \mathrm{O}_{2}(200 \mu \mathrm{mol} / \mathrm{L})$ 反应 $1 \mathrm{~h}$ 后 545 $\mathrm{nm}$ 处的 $I / I_{0}\left(I_{0}\right.$ 为探针在二甲基亚砜中的苂光强度)

Figure 1 (A) Fluorescence spectra of TPE-Py in DMSO/PBS mixtures with different volume fractions of $\mathrm{H}_{2} \mathrm{O}$ from 0 to $100 \%$ and (B) relative fluorescent intensity at $545 \mathrm{~nm}\left(I / I_{0}\right)$ of the probe $1(10 \mu \mathrm{mol} / \mathrm{L})$ after reaction with $\mathrm{H}_{2} \mathrm{O}_{2}(200 \mu \mathrm{mol} / \mathrm{L})$ for $1 \mathrm{~h}\left(I_{0}\right.$ is the fluorescent intensity of probe in DMSO)

$\mathrm{H}_{2} \mathrm{~S}$ )等的荧光光谱. 如图 $2 \mathrm{C}$ 所示, 除了 $\mathrm{KO}_{2} 、 t-\mathrm{BuOOH}$ 对探针有一定程度响应外, 其它均基本不会与探针反 应，表明探针对 $\mathrm{H}_{2} \mathrm{O}_{2}$ 具有较高的选择性. 由于弱碱性环 

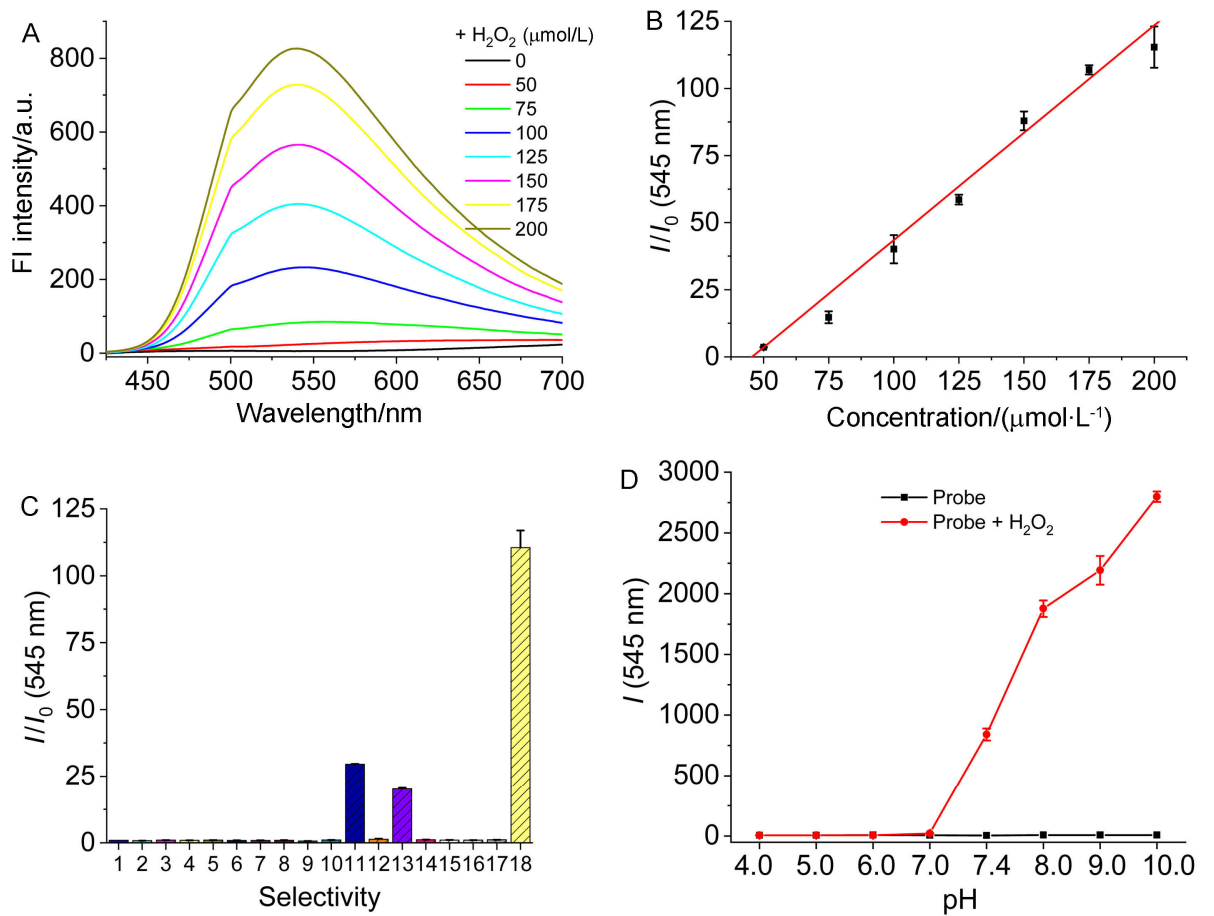

图 2 (A) 探针 1 在不同浓度 $\mathrm{H}_{2} \mathrm{O}_{2}$ 条件下的荧光光谱、(B)探针与 $\mathrm{H}_{2} \mathrm{O}_{2}$ 反应后, $545 \mathrm{~nm}$ 处荧光强度 $\left(I / I_{0}\right)$ 与 $\mathrm{H}_{2} \mathrm{O}_{2}$ 浓度拟合曲线、(C) 探针 $\mathbf{1}$ 的选择性曲线和(D)不同 $\mathrm{pH}$ 溶液中探针 $\mathbf{1}$ 与 $\mathrm{H}_{2} \mathrm{O}_{2}$ 条件反应前后的苂光强度(激发光 $405 \mathrm{~nm}$ ，反应时间 $1 \mathrm{~h}$ )

Figure 2 (A) Fluorescence spectra of probe response to different concentrations of $\mathrm{H}_{2} \mathrm{O}_{2}$, (B) relative fluorescent intensity at $545 \mathrm{~nm}$ $\left(I / I_{0}\right)$ as a function of $\mathrm{H}_{2} \mathrm{O}_{2}$ concentration, (C) selectivity of the probe towards different interfering species and (D) fluorescent intensity of the probe before and after reaction with $\mathrm{H}_{2} \mathrm{O}_{2}$ with different $\mathrm{pH}$ value (Excitation wavelength is $405 \mathrm{~nm}$, and the reacting time is $1 \mathrm{~h}$ ) In (C): 1 , probe 1 only; $2, \mathrm{Cu}^{2+} ; 3, \mathrm{Mg}^{2+} ; 4, \mathrm{Zn}^{2+} ; 5, \mathrm{Fe}^{3+} ; 6, \mathrm{NO}_{2}^{-} ; 7, \mathrm{SO}_{4}^{2-} ; 8, \mathrm{NO}_{3}^{-} ; 9, \mathrm{HSO}_{3}^{2-} ; 10, \mathrm{NaClO} ; 11, \mathrm{KO}_{2} ; 12, \mathrm{ONOO}^{-} ; 13, t-\mathrm{BuOOH}$; $14, \mathrm{NO} ; 15, \mathrm{GSH} ; 16$, Cys; $17, \mathrm{H}_{2} \mathrm{~S} ; 18, \mathrm{H}_{2} \mathrm{O}_{2}$

境更利于过氧化氢与嗍酸的反应 ${ }^{[34]}$, 测试了不同 $\mathrm{pH}$ 溶 液中探针与 $\mathrm{H}_{2} \mathrm{O}_{2}$ 反应的荧光光谱. 苂光光谱表明, 探针 可以用于中性和碱性条件下对 $\mathrm{H}_{2} \mathrm{O}_{2}$ 进行识别检测(图 2D).

\section{2 探针对过氧化氢的识别机制}

为了探究探针与 $\mathrm{H}_{2} \mathrm{O}_{2}$ 的识别机制, 测试了探针与 $\mathrm{H}_{2} \mathrm{O}_{2}$ 反应随时间变化的紫外可见吸收光谱和产物的核 磁共振氢谱. 如图 3 所示, 随着反应进行, 四苯乙烯吡 啶盐在 $420 \mathrm{~nm}$ 处的最大吸收峰发生明显蓝移. 同时, 在 紫外灯照射下，探针本身没有荧光，其与 $\mathrm{H}_{2} \mathrm{O}_{2}$ 反应后， 溶液呈现明显的黄色荧光(图 3 插图). 核磁共振氢谱显 示与 TPE-Py 一致. 推测该探针的识别机制与已报道文 献一致 ${ }^{[23]}: \mathrm{H}_{2} \mathrm{O}_{2}$ 首先氧化苯硼酸, 进而水解脱除硼酸, 随后中间体发生重排, 消除 4-亚甲基苯醌, 最终生成 TPE-Py，释放探针荧光(Scheme 2, B).

\section{3 探针对生物样本内过氧化氢的响应}

将探针 1 用于 $\mathrm{HeLa}$ 细胞中 $\mathrm{H}_{2} \mathrm{O}_{2}$ 荧光成像研究. 如 图 4 所示, 向细胞中单独加入探针 $(10 \mu \mathrm{mol} / \mathrm{L})$, 孵育 30 $\min$, 细胞呈现微弱的黄色苂光 (图 4A); 先捊育探针 30 $\min$, 再分别加入 $\mathrm{H}_{2} \mathrm{O}_{2}$ 继续孵育 $30 \mathrm{~min}$, 细胞黄色苂光 明显增强(图 4B, 4C). 结果表明, 探针具有较好的细胞

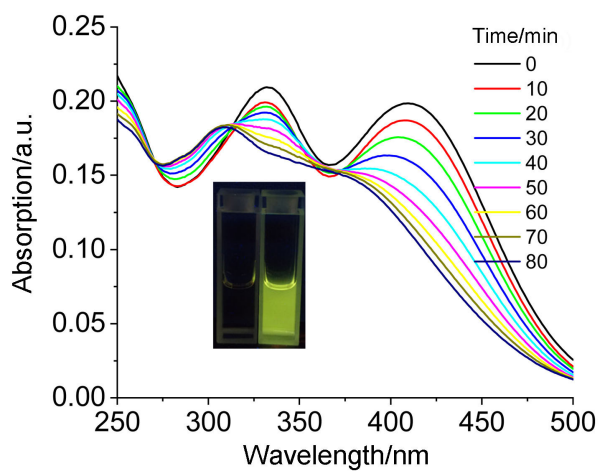

图 3 探针 $\mathbf{1}(10 \mu \mathrm{mol} / \mathrm{L})$ 与 $\mathrm{H}_{2} \mathrm{O}_{2}(200 \mu \mathrm{mol} / \mathrm{L})$ 反应溶液的紫外 反应动力学曲线

Figure 3 UV-Vis kinetic curves of probe $1(10 \mu \mathrm{mol} / \mathrm{L})$ and $\mathrm{H}_{2} \mathrm{O}_{2}(200 \mu \mathrm{mol} / \mathrm{L})$

Inset: color changes of probe $\mathbf{1}$ in the absence and presence of $\mathrm{H}_{2} \mathrm{O}_{2}$ under UV light (365 nm)

通透性，可以用于细胞内 $\mathrm{H}_{2} \mathrm{O}_{2}$ 成像研究.

缺血再灌注是组织缺血或缺氧一段时间后供血恢 复的临床高发现象，普遍存在于外科手术、器官移植、 创伤性休克和血栓等多种血液循环障碍过程. 研究表明 缺血组织再灌注会导致细胞产生大量 ROS，从而对微 血管和实质器官造成损伤. 为了模拟在体的缺血缺氧状 态，构建了细胞氧糖剥离再灌注 $(\mathrm{OGD} / \mathrm{R})$ 模型 ${ }^{[35]}$. 分别 


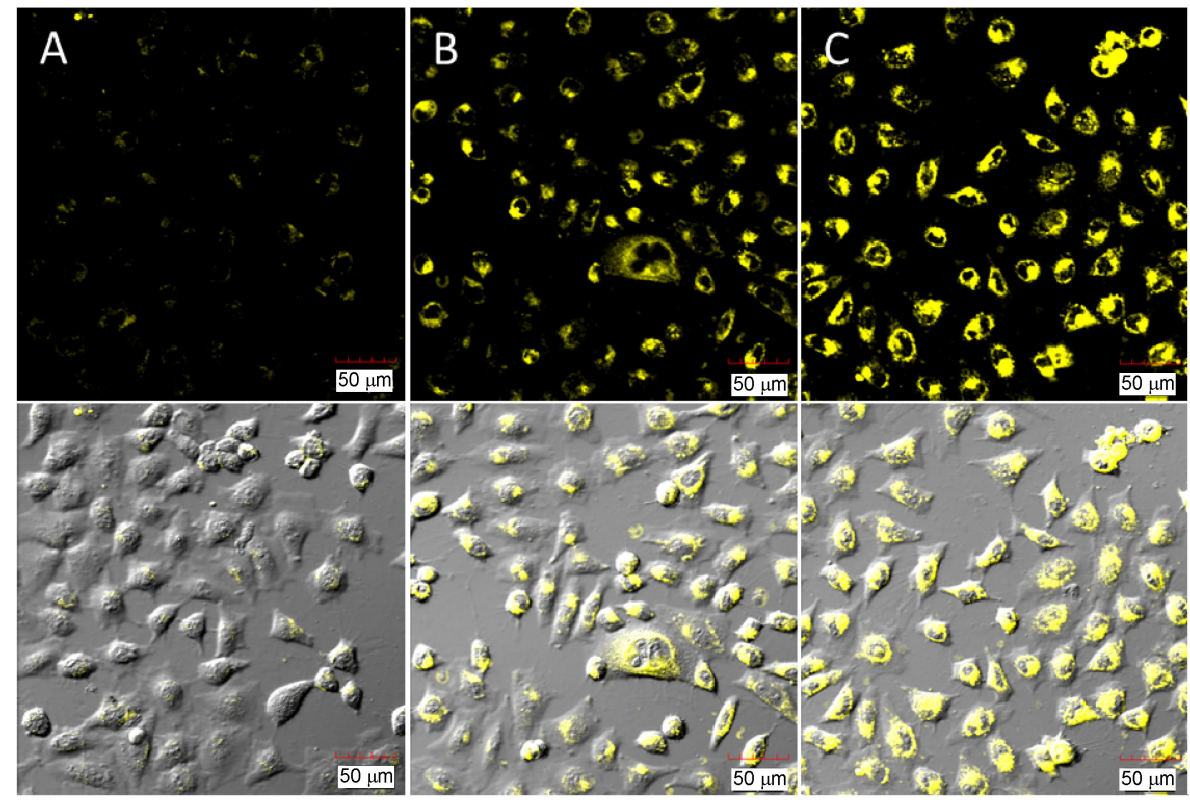

图 4 细胞外源 $\mathrm{H}_{2} \mathrm{O}_{2}$ 苂光共聚焦成像

Figure 4 Fluorescence confocal imaging of exogenous $\mathrm{H}_{2} \mathrm{O}_{2}$

(A) Images of HeLa cells treated with only probe for $30 \mathrm{~min}$; (B and C) images of cells which was firstly treated with probe for 30 min, then incubated with different concentration of $\mathrm{H}_{2} \mathrm{O}_{2}$ (B: $100 \mu \mathrm{mol} / \mathrm{L}, \mathrm{C}: 200 \mu \mathrm{mol} / \mathrm{L}$ ) for $30 \mathrm{~min}$. Up: fluorescent fields; Down: merged images. Scale bars $=50 \mu \mathrm{m}$

向正常培养和 $\mathrm{OGD} / \mathrm{R}$ 模型 $\mathrm{HeLa}$ 细胞中加入探针(10 $\mu \mathrm{mol} / \mathrm{L})$, 孵育 $30 \mathrm{~min}$, 发现 OGD/R 模型 HeLa 细胞的黄 色苂光明显增强(图 5), 表明缺血再灌注过程中可以诱 导大量 $\mathrm{H}_{2} \mathrm{O}_{2}$ 生成.

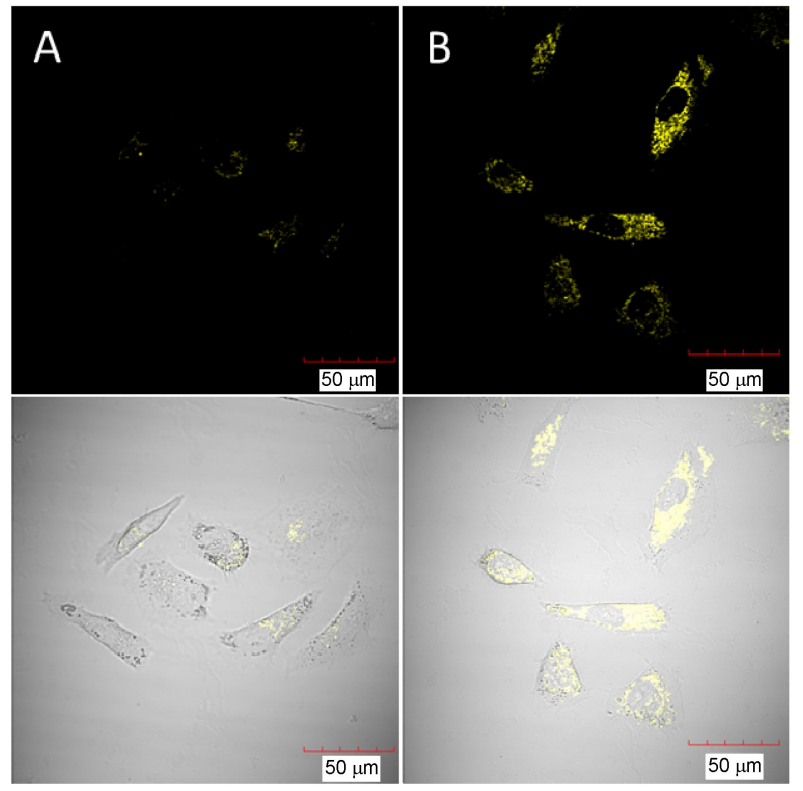

图 5 氧糖剥离再灌注诱导细胞内源 $\mathrm{H}_{2} \mathrm{O}_{2}$ 苂光共聚焦成像

Figure 5 Fluorescence confocal imaging of OGD/R induced endogenous $\mathrm{H}_{2} \mathrm{O}_{2}$

(A) Images of only probe treated HeLa cells; (B) Images of only probe treated OGD/R model HeLa cells. Up: fluorescent fields, Down: merged images. Scale bars $=50 \mu \mathrm{m}$

斑马鱼作为模式生物, 被用于苂光共聚焦成像研
究. 如图 6 所示，斑马鱼本身并没有显示出苂光(图 6A), 向培养液中加入探针 $(10 \mu \mathrm{mol} / \mathrm{L})$ 培养 $30 \mathrm{~min}$ 后, 斑马鱼 卵黄和肠球等部位呈现出微弱的绿色苂光(图 6B), 再加 入 $\mathrm{H}_{2} \mathrm{O}_{2}(100 \mu \mathrm{mol} / \mathrm{L})$ 继续孵育 $30 \mathrm{~min}$ 后, 斑马鱼相应部 位绿色菼光明显增强(图 6C). 说明, 探针具有较好的细 胞通透性. 据报道, 脂多糖(LPS) 可以诱导组织产生炎 症 $^{[36]}$. 向培养液中加入 LPS $\left(1 \times 10^{-6} \mathrm{~g} / \mathrm{mL}\right)$ 培养 $12 \mathrm{~h}$, 再加入探针 $(10 \mu \mathrm{mol} / \mathrm{L})$ 孵育 $30 \mathrm{~min}$ 后, 斑马鱼卵黄和肠 球等部位出现明显的绿色苂光(图6D), 表明 LPS 诱导炎 症过程伴随大量 $\mathrm{H}_{2} \mathrm{O}_{2}$ 的产生. 以上成像实验结果表明, 探针具有良好的组织渗透性，可以用于外源刺激物诱导 斑马鱼内源 $\mathrm{H}_{2} \mathrm{O}_{2}$ 的苂光成像研究.

\section{2 结论}

合成了具有长发射波长的聚集诱导发光苂光探针, 实现了对 $\mathrm{H}_{2} \mathrm{O}_{2}$ 的打开型荧光检测. 该探针对 $\mathrm{H}_{2} \mathrm{O}_{2}$ 具有 良好的选择性和灵敏性，可以联合葡萄糖氧化酶对 $D$ 葡萄糖选择性检测. 此外, 探针具有良好的细胞和组织 穿透性, 可以用于氧糖剥离再灌注诱导 HeLa 细胞和 LPS 诱导斑马鱼内源 $\mathrm{H}_{2} \mathrm{O}_{2}$ 成像研究.

\section{3 实验部分}

\section{1 实验仪器和材料}

Brucker 核磁共振仪(400 MHz)、Hitachi F-7000 型苂 光光谱仪(日本 Hitachi 公司)、UV-3600 型分光光度计(美 


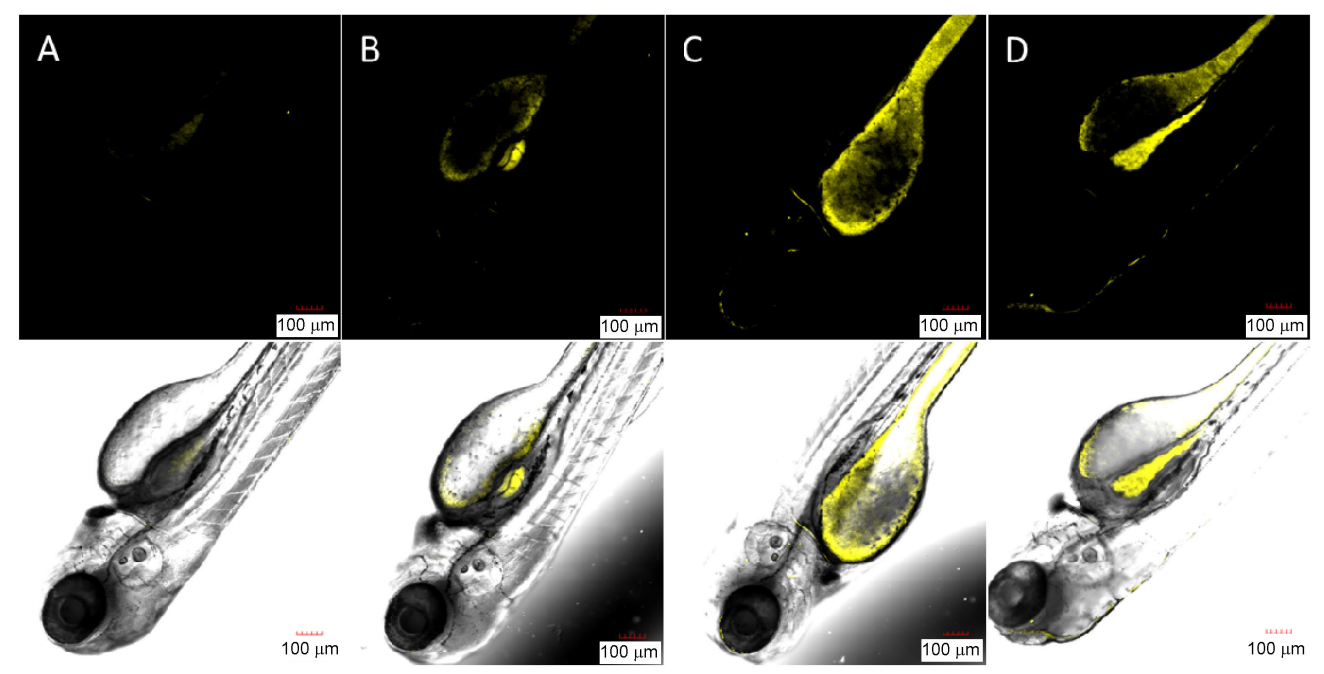

图 6 探针 1 的斑马鱼苂光共聚焦成像

Figure 6 Fluorescence confocal imaging of probe 1

(A) Images of only zebrafish; (B) images of only probe treated zebrafish; (C) images of zebrafish which was firstly treated with probe, then incubated with $\mathrm{H}_{2} \mathrm{O}_{2}$; (D) images of zebrafish which was firstly treated with LPS, then incubated with probe. Up: fluorescent fields; Down: merged images. Scale bars= $100 \mu \mathrm{m}$

国 Agilent 公司)、倒置苂光共聚焦显微镜(FV1000-ASV, 日本奥林巴斯)及精密 pH 计(HANNA, HI8424).

4-澳二苯酮、4,4'-二甲氧基二苯酮、 $\mathrm{TiCl}_{4} 、 \mathrm{Zn}$ 粉、 4-乙烯基吡啶、 $\mathrm{Pd}(\mathrm{OAc})_{2}$ 、邻甲苯基膦、 $N, N$-二异丙基 乙胺(DIPEA)等试剂均购自萨恩化学技术(上海)有限公 司; 过氧化氢为 $30 \%$ (质量分数) 分析纯(上海沃凯生物技 术有限公司); $N, N$-二甲基甲酰胺(DMF)、DMSO、乙酸 乙酯、二氯甲烷和甲醇均为分析纯, 购自国药集团化学 试剂有限公司. 柱层析用硅胶(100 200 目)及 GF254 薄 层层析硅胶为青岛海洋化工厂产品.

\section{2 探针的合成}

将 $100.0 \mathrm{mg}$ TPE-Py $(0.200 \mathrm{mmol})$ 和 $46.0 \mathrm{mg} 4$-溴甲 基苯硼酸 $(0.210 \mathrm{mmol})$ 溶解于 $4.0 \mathrm{~mL}$ 乙二醇单甲醚中, $85{ }^{\circ} \mathrm{C}$ 反应过夜. 减压蒸除乙二醇单甲醚, 以二氯甲烷 和甲醇 $(V: V=50: 1)$ 为淋洗剂柱色谱分离, 得 $80 \mathrm{mg}$ 红色固体, 产率 $56.4 \%$. m.p. $56.8 \sim 58{ }^{\circ} \mathrm{C} ;{ }^{1} \mathrm{H}$ NMR (DMSO- $\left.d_{6}, 400 \mathrm{MHz}\right) \delta: 3.68(\mathrm{~s}, 6 \mathrm{H}), 5.74(\mathrm{~s}, 1 \mathrm{H}), 5.76(\mathrm{~s}$, $1 \mathrm{H}), 6.74 \sim 6.67(\mathrm{~m}, 2 \mathrm{H}), 6.92 \sim 6.84(\mathrm{~m}, 2 \mathrm{H}), 7.00 \sim 6.96$ (m, 1H), $7.04(\mathrm{~d}, J=8.3 \mathrm{~Hz}, 1 \mathrm{H}), 7.20 \sim 7.09(\mathrm{~m}, 2 \mathrm{H})$, 7.44 (dd, $J=12.2,7.9 \mathrm{~Hz}, 2 \mathrm{H}), 7.52(\mathrm{~d}, J=8.3 \mathrm{~Hz}, 1 \mathrm{H})$, 7.84 (d, $J=8.0 \mathrm{~Hz}, 1 \mathrm{H}), 7.92$ (d, $J=16.2 \mathrm{~Hz}, 1 \mathrm{H}), 8.14$ (s, $1 \mathrm{H}), 8.20(\mathrm{~d}, J=6.4 \mathrm{~Hz}, 1 \mathrm{H}), 9.03(\mathrm{~d}, J=5.9 \mathrm{~Hz}, 1 \mathrm{H}) ;{ }^{13} \mathrm{C}$ NMR (DMSO- $\left.d_{6}, 100 \mathrm{MHz}\right) \delta$ : 55.20, 55.38, 62.82, $113.63,113.77,124.49,128.01,128.29,128.48,131.22$, $131.94,132.48,132.56,135.28,135.89,136.48,138.55$, 144.59, 146.92, 158.40; HRMS calcd for $\mathrm{C}_{42} \mathrm{H}_{37} \mathrm{NBO}_{4}$ 630.2810 , found 630.2816 .

\section{3 光谱测试}

探针及其对照分子用 DMSO 配制成 $4.0 \times 10^{-3}$ $\mathrm{mol} / \mathrm{L}$ 的母液, 测试浓度为 $10 \mu \mathrm{mol} / \mathrm{L}$. 选择性测试所用 各种干扰离子均为钠盐或钾盐，除 Cys、Hcy 测试浓度 为 $1.0 \times 10^{-3} \mathrm{~mol} / \mathrm{L}, \mathrm{GSH}$ 浓度为 $5.0 \times 10^{-3} \mathrm{~mol} / \mathrm{L}$ 外, 其 它干扰离子测试浓度为 $1.0 \times 10^{-4} \mathrm{~mol} / \mathrm{L}$, 测试溶液为磷 酸盐缓冲液 $\left(\mathrm{PBS}, 1.0 \times 10^{-2} \mathrm{~mol} / \mathrm{L}, \mathrm{pH} \mathrm{7.4}\right.$, 体积分数为 $60 \%$ 的 DMSO). 苂光光谱、紫外光谱均在 $25{ }^{\circ} \mathrm{C}$ 条件下 测试, 孵育时间为 $60 \mathrm{~min}$, 样品池为 $1 \mathrm{~cm} \times 1 \mathrm{~cm} \times 4 \mathrm{~cm}$ 石英比色血, 激发波长为 $365 \mathrm{~nm}$, 激发和发射狭缝宽度 均为 $5 \mathrm{~nm}$.

\section{4 细胞实验}

细胞培养: HeLa 细胞在高糖培养基(含有体积分数 为 $10 \%$ 的胎牛血清、 $100 \mathrm{U} / \mathrm{mL}$ 盘尼西林、 $100 \mathrm{mg} / \mathrm{mL}$ 链霉素和 $4 \times 10^{-3} \mathrm{~mol} / \mathrm{L} L$-谷氨酰胺), 体积分数为 $5 \%$ 的 $\mathrm{CO}_{2}, 37{ }^{\circ} \mathrm{C}$ 条件下培养.

$\mathrm{OGD} / \mathrm{R}$ 细胞模型建立: 用 PBS 洗涤 HeLa 细胞三次, 将其加入无糖培养基和连二硫酸钠 $\left(0.5 \times 10^{-3} \mathrm{~mol} / \mathrm{L}\right)$ 中 培养 $30 \mathrm{~min}$. 随后将细胞转移至高糖培养基, 体积分数 为 $5 \%$ 的 $\mathrm{CO}_{2}, 37{ }^{\circ} \mathrm{C}$ 条件下捊育 $30 \mathrm{~min}$.

细胞成像实验: HeLa 细胞接种于成像专用玻底培 养血, 密度为 $2 \times 10^{4}\left(/ \mathrm{cm}^{-2}\right)$. 探针组仅加入探针 $1(10$ $\mu \mathrm{mol} / \mathrm{L}$ ) 孵育 $30 \mathrm{~min}$; 外源 $\mathrm{H}_{2} \mathrm{O}_{2}$ 成像组先加入探针 1 $(10 \mu \mathrm{mol} / \mathrm{L})$ 稃育 $30 \mathrm{~min}, \mathrm{PBS}$ 洗涤三次去除过量探针后, 再分别加入 $\mathrm{H}_{2} \mathrm{O}_{2}(100,200 \mu \mathrm{mol} / \mathrm{L})$ 继续孵育 $30 \mathrm{~min}$, 进 行荧光成像. $\mathrm{OGD} / \mathrm{R}$ 模型组加入探针 $1(10 \mu \mathrm{mol} / \mathrm{L})$, 孵 育 $30 \mathrm{~min}$; PBS 洗涤三次去除过量探针, 进行荧光成像. 
共聚焦苂光成像激发波长为 $405 \mathrm{~nm}$, 收集 495 595 nm 范围的图像.

\section{5 斑马鱼实验}

3 日龄斑马鱼用于苂光成像. 对照组不对斑马鱼进 行任何处理; 探针组仅加入探针 $1(10 \mu \mathrm{mol} / \mathrm{L})$ 餒育 30 $\mathrm{min}$; 外源 $\mathrm{H}_{2} \mathrm{O}_{2}$ 成像组先加入探针 $\mathbf{1}(10 \mu \mathrm{mol} / \mathrm{L})$ 孵育 30 min, PBS 洗涤三次去除过量探针后, 再加入 $\mathrm{H}_{2} \mathrm{O}_{2}(100$ $\mu \mathrm{mol} / \mathrm{L}$ )继续孵育 $30 \mathrm{~min}$; LPS 诱导内源 $\mathrm{H}_{2} \mathrm{O}_{2}$ 成像组先 加入 LPS $\left(1 \times 10^{-6} \mathrm{~g} / \mathrm{mL}\right)$ 孵育 $12 \mathrm{~h}$, 再加入探针 $1(10$ $\mu \mathrm{mol} / \mathrm{L}$ )继续孵育 $30 \mathrm{~min}, \mathrm{PBS}$ 洗涤三次去除过量探针 后，进行荧光成像. 共聚焦荧光成像激发波长为 405 $\mathrm{nm}$, 收集 495 595 nm 范围的图像.

辅助材料(Supporting Information) 化合物 TPE-Py 和 探针 1 的 ${ }^{1} \mathrm{H}$ NMR, ${ }^{13} \mathrm{C}$ NMR 以及 HRMS 谱图, 探针的 部分光谱测试等。这些材料可以免费从本刊网站 (http://sioc-journal.cn/)上下载.

\section{References}

[1] Rhee, S. G. Science 2006, 312, 1882.

[2] Mishina, N. M.; Tyurin-Kuzmin, P. A.; Markvicheva, K. N.; Vorotnikov, A. V.; Tkachuk, V. A.; Laketa, V.; Schultz, C.; Lukyanov, S.; Belousov, V. V. Antioxid. Redox Signaling 2011, 15, 1.

[3] Hurd, T. R.; DeGennaro, M.; Lehmann, R. Trends Cell Biol. 2012, $22,107$.

[4] Ohshima, H.; Tatemichi, M.; Sawa, T. Arch. Biochem. Biophys 2003, 417, 3.

[5] Silva, G. F.; Ming, L. J. Angew. Chem., Int. Ed. 2007, 46, 3337.

[6] Barnham, K. J.; Masters, C. L.; Bush, A. I. Nat. Rev. Drug Discovery 2004, 3, 205.

[7] Gottlieb, R. A.; Burleson, K. O.; Kloner, R. A.; Babior, B. M.; Engler, R. L. J. Clin. Invest. 1994, 94, 1621.

[8] Li, L.; Zhang, Y.; Zhang, L. N.; Ge, S. G.; Liu, H. Y.; Ren, N.; Yan, M.; Yu, J. H. Anal. Chem. 2016, 88, 5369.

[9] Matsubara, C.; Kawamoto, N.; Takamura, K. Analyst 1992, 117, 1781 .

[10] Yang, S. M.; Huang, A.-H.; Cha, W. L.; Wei, Z. P.; Zheng, L, Z. Acta Chim. Sinica 2011, 69, 2143 (in Chinese). (杨绍明，黄爱花，查文玲，魏志鹏，郑龙珍，化学学报, 2011，69, 2143.)

[11] Li, Z. Z.; Xin, Y. M.; Wu, W. L.; Fu, B. L.; Zhang, Z. H. Anal. Chem. 2016, 88, 7724

[12] Lippert, A. R.; Bittner, G. C.; Chang, C. J. Acc. Chem. Res. 2011,
44, 793

[13] Andina, D.; Leroux, J.-C.; Luciani, P. Chem.-Eur. J. 2017, 23, 13549

[14] Rezende, F.; Brandes, R. P.; Schroeder, K. Antioxid. Redox Signaling 2018, 29, 585.

[15] Liu, Y.; Jiao, C.; Lu, W.; Zhang, P.; Wang, Y. RSC Adv. 2019, 9, 18027

[16] Chang, M. C.; Pralle, A.; Isacoff, E. Y.; Chang, C. J. J. Am. Chem. Soc. 2004, 126, 15392.

[17] Chung, C.; Srikun, D.; Lim, C. S.; Chang, C. J.; Cho, B. R. Chem Commun. 2011, 47, 9618.

[18] Ang, C. Y.; Tan, S. Y.; Wu, S. J.; Qu, Q. Y.; Wong, M. F. E.; Luo, Z.; Li, P. Z.; Selvan, S. T.; Zhao, Y. L. J. Mater. Chem. C 2016, 4 , 2761.

[19] Liu, J.; Ren, J.; Bao, X. J.; Gao, W.; Wu, C. L.; Zhao, Y. B. Anal. Chem. 2016, 88,5865 .

[20] Ren, M. G.; Deng, B. B.; Wang, J. Y.; Kong, X. Q.; Liu, Z. R.; Zhou, K.; He, L. W.; Lin, W. Y. Biosens. Bioelectron. 2016, 79, 237.

[21] Xiao, H.; Li, P.; Hu, X.; Shi, X.; Zhang, W.; Tang, B. Chem. Sci. 2016, 7, 6153 .

[22] Liu, X.; He, L.; Yang, L.; Geng, Y.; Yang, L.; Song, X. Sens. Actuators, $B$ 2018, 259, 803

[23] Shen, Y.; Zhang, X.; Zhang, Y.; Wu, Y.; Zhang, C.; Chen, Y.; Jin, J.; Li, H. Sens. Actuators, B 2018, 255, 42.

[24] Zhu, B.; Wu, L.; Wang, Y.; Zhang, M.; Zhao, Z.; Liu, C.; Wang, Z.; Duan, Q.; Jia, P. Sens. Actuators, B 2018, 259, 797.

[25] Zhang, J.; Shi, L.; Li, Z.; Li, D.; Tian, X.; Zhang, C. Analyst 2019 , $144,3643$.

[26] Abo, M.; Urano, Y.; Hanaoka, K.; Terai, T.; Komatsu, T.; Nagano, T. J. Am. Chem. Soc. 2011, 133, 10629.

[27] Zhang, K. M.; Dou, W.; Li, P. X.; Shen, R.; Ru, J. X.; Liu, W.; Cui, Y. M.; Chen, C. Y.; Liu, W. S.; Bai, D. C. Biosens. Bioelectron. 2015, 64, 542 .

[28] Xie, X.; Yang, X. E.; Wu, T.; Li, Y.; Li, M.; Tan, Q.; Wang, X.; Tang, B. Anal. Chem. 2016, 88, 8019.

[29] Peng, J.; Hou, X.; Zeng, F.; Wu, S. Biosens. Bioelectron. 2017, 94 278.

[30] Luo, J. D.; Xie, Z. L.; Zhu, D. B.; Tang, B. Z. Chem. Commun. 2011, $18,1740$.

[31] Zhang, J.; Wang, Q.; Guo, Z.; Zhang, S.; Yan, C.; Tian, H.; Zhu, W. Adv. Funct. Mater. 2019, 29, 1808153.

[32] Zhang, W.; Liu, W.; Li, P.; Huang, F.; Wang, H.; Tang, B. Anal. Chem. 2015, 87, 9825

[33] Hu, F.; Huang, Y.; Zhang, G.; Zhao, R.; Zhang, D. Tetrahedron Lett 2014, 55, 1471.

[34] Xu, J.; Zhang, Y.; Yu, H.; Gao, X.; Shao, S. Anal. Chem. 2016, 88, 1455.

[35] Li, Y.; Wang, X.; Yang, J.; Xie, X. L.; Li, M. M.; Niu, J. Y.; Tong, L. L.; Tang, B. Anal. Chem. 2016, 88, 11154

[36] Kumar, R.; Han, J.; Lim, H.-J.; Ren, W. X.; Lim, J.-Y.; Kim, J.-H.; Kim, J. S. J. Am. Chem. Soc. 2014, 136, 17836. 